\title{
A multi-epoch spectrophotometric atlas of symbiotic stars ${ }^{\star, \star \star, \star \star \star}$
}

\author{
U. Munari ${ }^{1,2}$ and T. Zwitter ${ }^{3}$ \\ 1 Osservatorio Astronomico di Padova, Sede di Asiago, 36032 Asiago (VI), Italy \\ 2 CISAS - Centro Inter-Dipartimentale per Studi ed Attività Spaziali, Univ. di Padova, Italy \\ 3 University of Ljubljana, Department of Physics, Jadranska 19, 1000 Ljubljana, Slovenia \\ e-mail: tomaz.zwitter@uni-lj.si
}

Received 9 October 20001 / Accepted 22 November 2001

\begin{abstract}
A multi-epoch, absolute-fluxed spectral atlas extending from about 3200 to $9000 \AA$ is presented for 130 symbiotic stars, including members of the LMC, SMC and Draco dwarf galaxies. The fluxes are accurate to better than 5\% as shown by comparison with Tycho and ground-based photometric data. The spectra of 40 reference objects (MKK cool giant standards, Mira and Carbon stars, planetary nebulae, white dwarfs, hot sub-dwarfs, Wolf-Rayet stars, classical novae, VV Cep and Herbig Ae/Be objects) are provided to assist the interpretation of symbiotic star spectra. Astrometric positions and counterparts in astrometric catalogues are derived for all program symbiotic stars. The spectra are available in electronic form from the authors.
\end{abstract}

Key words. stars: binaries: symbiotic - atlases

\section{Introduction}

Allen's (1984) atlas offered the first comprehensive spectroscopic view of symbiotic stars. It included spectra covering the 3400-7500 $\AA$ range for 114 objects (validated and possible, corresponding to $72 \%$ of the total number known at the time), which however were not fluxed, of low resolution and low dynamic range. Nevertheless, Allen's catalogue has been extensively used in all studies of symbiotic stars, even in those concerning single objects, because its single-epoch spectra could be compared with later observations in order to study the marked spectral variability of these binaries.

Other compilations of symbiotic star optical spectra are available. The larger ones since Allen's atlas are Blair et al. (1983; 16 objects, absolute fluxes), Ipatov \& Yudin (1986; 14, absolute), Kenyon \& Fernandez-Castro (1987; 11, absolute), Acker et al. (1988; 10, relative),

Send offprint requests to: U. Munari,

e-mail: munari@astras.pd.astro.it

* Based on observations collected with the telescopes of the European Southern Observatory (ESO, Chile) and of the Padova \& Asiago Astronomical Observatories (Italy).

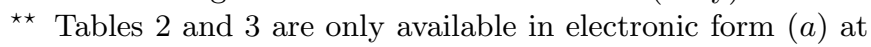
the CDS via anonymous ftp to

cdsarc.u-strasbg.fr (130.79.128.5) or via

http://cdsweb.u-strasbg.fr/cgi-bin/qcat?J/A+A/383/188, and $(b)$ from the personal home page

http://ulisse.pd.astro.it/symbio_atlas/

$\star \star \star$ Figures 4-256 are only available in electronic form $(a)$ at http://www.edpsciences.org and (b) from the personal home page http://ulisse.pd.astro.it/symbio_atlas/
Múrset et al. (1996; 12, absolute), Gutierrez-Moreno et al. (1999; 14, absolute) and Medina Tanco \& Steiner (1995; 45, relative). Meier et al. (1994) assembled an atlas of ultraviolet IUE spectra for 32 symbiotic stars, SchulteLadbeck (1988) of 16 objects in the near-IR and Schild et al. (1992) of 8 objects in the IR. Van Winckel et al. (1993) surveyed emission line profiles for 59 objects and Ivison et al. (1994) for 35. Pereira et al. (1999) presented Bowen-fluorescence dominated blue spectra for 8 symbiotic stars and Schmid \& Schild (1994) surveyed Ramanscatter dominated red spectra for 15 objects.

Here we present the largest optical spectroscopic atlas of symbiotic stars since Allen's one. Compared to Allen's atlas, our spectra cover a comparable number of objects (130 in all, 75 observed from ESO, 40 from Asiago, and 15 from both places) but are better resolved, extend over a wider wavelength range, offer a higher dynamical range, are absolutely fluxed, are multi-epoch (half of the targets re-observed one or more times over a three year period), and are available in electronic form (from the authors upon request).

The spectra presented in this atlas are planned to become the input data for future follow-up studies:

- given the high accuracy of the absolute fluxes and the wide wavelength range covered, optical magnitudes will be derived from the spectra and combined into a unique multi-epoch $U B V(R I)_{\mathrm{C}}$ photometric catalogue with the results of CCD photometry of 60 symbiotic stars obtained by Henden \& Munari (2000, 2001, 2002) while calibrating their comparison sequences; 
RR Tel

22.33/04/95

ESO $1.5 \mathrm{~m}+\mathrm{B \& C}$
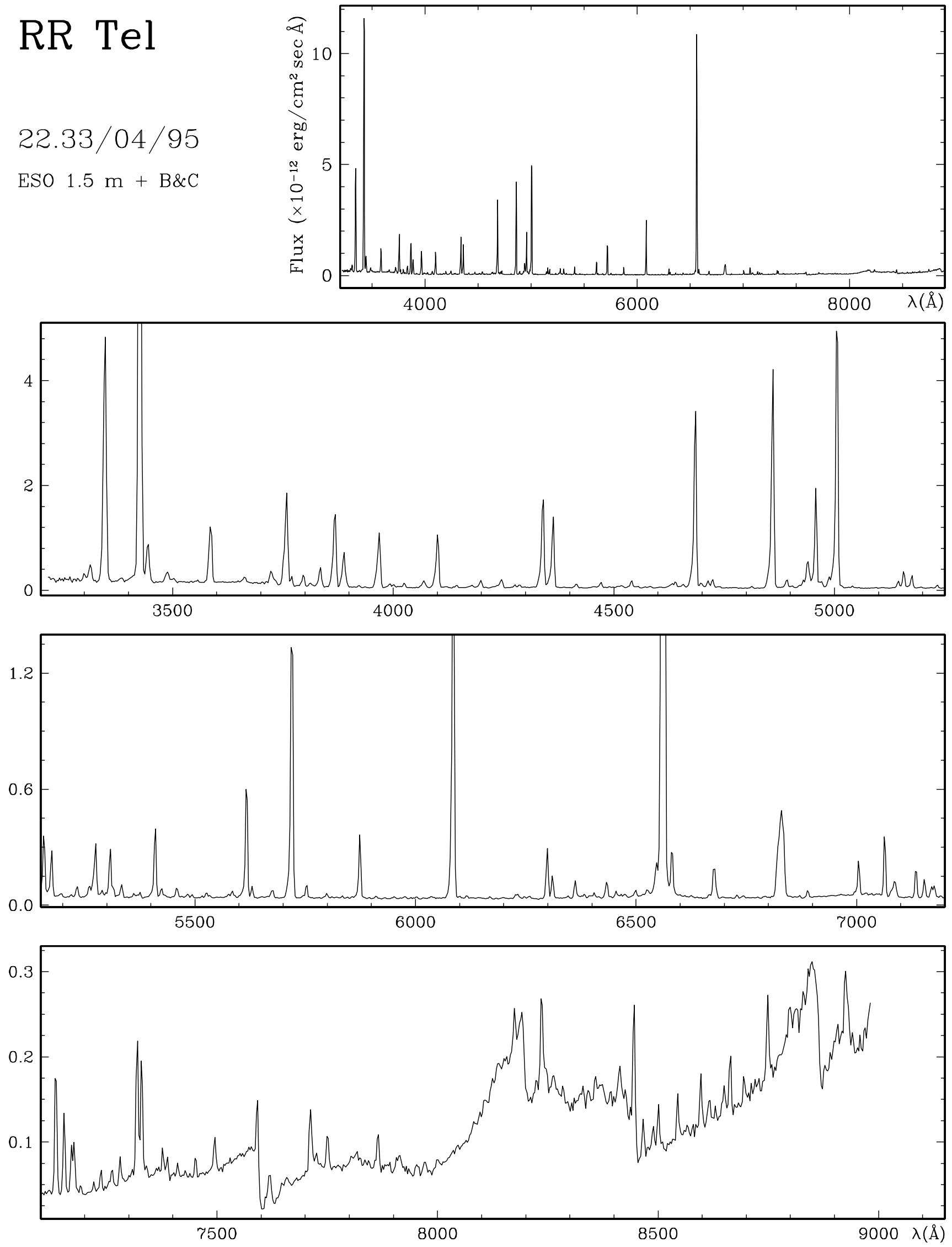

Fig. 1. The spectrum of the symbiotic star RR Tel. This is an example for the Figs. 4-256 available electronically only. 
Table 1. List of program stars and the journal of observations, separately for ESO and Asiago observations.

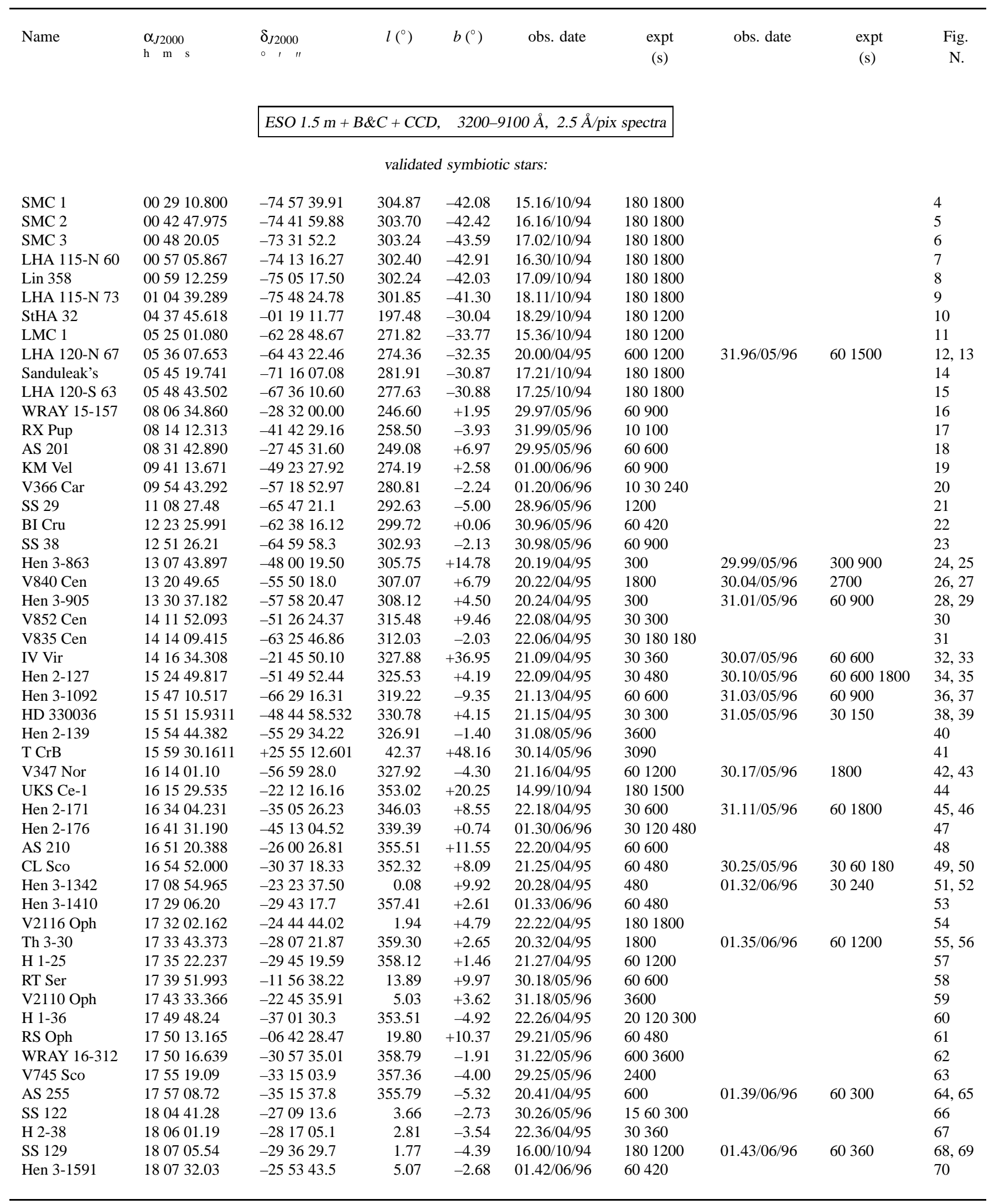


Table 1. continued.

\begin{tabular}{|c|c|c|c|c|c|c|c|c|c|}
\hline Name & $\begin{array}{l}\alpha_{J 2000} \\
\mathrm{~h} \quad \mathrm{~m} s\end{array}$ & $\begin{array}{c}\delta_{J 2000} \\
\circ, "\end{array}$ & $l\left(^{\circ}\right)$ & $b\left(^{\circ}\right)$ & obs. date & $\begin{array}{l}\text { expt } \\
\text { (s) }\end{array}$ & obs. date & $\begin{array}{l}\text { expt } \\
\text { (s) }\end{array}$ & $\begin{array}{l}\text { Fig } \\
\mathrm{N} .\end{array}$ \\
\hline YY Her & 181434.207 & +205921.02 & 48.14 & +17.24 & $01.28 / 06 / 96$ & 30150420 & & & 71 \\
\hline FG Ser & 181507.096 & -001852.21 & 28.48 & +7.93 & $29.34 / 05 / 96$ & 15180 & & & 72 \\
\hline Hen 3-1674 & 182019.15 & -262247.0 & 6.01 & -5.43 & $31.42 / 05 / 96$ & 600 & 01.38/06/96 & 900 & 73,74 \\
\hline V3929 Sgr & 182058.851 & -264825.09 & 5.70 & -5.76 & $22.39 / 04 / 95$ & 6001080 & $31.28 / 05 / 96$ & 1200 & 75,76 \\
\hline V3811 Sgr & 182328.955 & -215309.62 & 10.34 & -3.98 & $31.37 / 05 / 96$ & 6001800 & & & 77 \\
\hline V4018 Sgr & 182526.845 & -283557.49 & 4.55 & -7.46 & $22.30 / 04 / 95$ & 30360 & & & 78 \\
\hline К 3-9 & 184024.117 & -084357.80 & 23.91 & -1.54 & $22.41 / 04 / 95$ & 300600 & & & 79 \\
\hline StHA 154 & 184327.928 & +193005.43 & 49.60 & +10.45 & $19.96 / 04 / 95$ & 30600 & & & 80 \\
\hline V4368 Sgr & 185440.32 & -194159.8 & 15.59 & -9.53 & $18.42 / 04 / 95$ & 150600 & 01.32/06/96 & 3090 & 81,82 \\
\hline V1413 Aql & 190351.685 & +162831.74 & 49.02 & +4.77 & $29.29 / 05 / 96$ & 60600 & & & 83 \\
\hline Ap 3-1 & 191036.126 & +024928.69 & 37.64 & -2.97 & $21.41 / 04 / 95$ & 1201080 & & & 84 \\
\hline HM Sge & 194157.083 & +164439.92 & 53.57 & -3.15 & $22.35 / 04 / 95$ & 10120 & & & 85 \\
\hline V1016 Cyg & 195705.027 & +394936.13 & 75.17 & +5.68 & $22.40 / 04 / 95$ & 51560 & & & 86 \\
\hline RR Tel & 200418.459 & -554332.27 & 342.16 & -32.24 & $22.33 / 04 / 95$ & 530 & & & 87 \\
\hline PU Vul & 202113.330 & +213419.31 & 62.58 & -8.52 & $21.38 / 04 / 95$ & 52060240 & $30.34 / 05 / 96$ & 1030150 & 88,89 \\
\hline StHA 176 & 202242.25 & -210754.9 & 22.65 & -29.10 & $18.05 / 10 / 94$ & 1801200 & & & 90 \\
\hline LT Del & 203557.244 & +201127.60 & 63.40 & -12.15 & $21.39 / 04 / 95$ & 30420 & & & 91 \\
\hline ER Del & 204246.504 & +084112.09 & 54.46 & -20.00 & $30.35 / 05 / 96$ & 80 & & & 92 \\
\hline V1329 Cyg & 205101.27 & +353454.1 & 77.80 & -5.56 & $29.37 / 05 / 96$ & 30420 & & & 93 \\
\hline $\mathrm{CD}-43.14304$ & 210006.38 & -423844.9 & 358.65 & -41.10 & $19.43 / 04 / 95$ & 30180 & $30.36 / 05 / 96$ & 5120 & 94,95 \\
\hline StHA 190 & 214144.891 & +024354.41 & 58.42 & -35.43 & $16.12 / 10 / 94$ & 30180600 & & & 96 \\
\hline AG Peg & 215101.9749 & +123732.113 & 69.28 & -30.89 & $29.39 / 05 / 96$ & 310 & & & 97 \\
\hline R Aqr & 234349.4616 & -151704.202 & 66.52 & -70.33 & $30.43 / 05 / 96$ & 1 & & & 98 \\
\hline
\end{tabular}

possible symbiotic stars or closely related objects

\begin{tabular}{|c|c|c|c|c|c|c|c|c|c|}
\hline Ma 51 & 004334.160 & -731324.67 & 303.72 & -43.89 & $15.19 / 10 / 94$ & 1801800 & & & 99 \\
\hline Ma 250 & 004843.194 & -730311.57 & 303.21 & -44.07 & $17.06 / 10 / 94$ & 1801800 & & & 100 \\
\hline Ma 285 & 004913.624 & -725428.96 & 303.16 & -44.22 & $18.08 / 10 / 94$ & 180 & & & 101 \\
\hline Мa 642 & 005304.092 & -721616.56 & 302.76 & -44.86 & $16.20 / 10 / 94$ & 1801800 & & & 102 \\
\hline Мa 832 & 005517.486 & -720310.92 & 302.52 & -45.08 & $16.23 / 10 / 94$ & 1801200 & & & 103 \\
\hline Мa 966 & 005652.98 & -722111.3 & 302.35 & -44.77 & $16.25 / 10 / 94$ & 1801800 & & & 104 \\
\hline Мa 1591 & 010632.257 & -721718.50 & 301.31 & -44.80 & $18.14 / 10 / 94$ & 1801800 & & & 105 \\
\hline Ma 1858 & 011835.682 & -724221.78 & 300.12 & -44.27 & $17.12 / 10 / 94$ & 1801800 & & & 106 \\
\hline StHA 55 & 054642.079 & +064347.41 & 199.34 & -11.12 & $19.24 / 10 / 94$ & 180 & & & 107 \\
\hline V704 Cen & 135455.738 & -582716.53 & 311.17 & +3.40 & $21.07 / 04 / 95$ & 1200 & $29.00 / 05 / 96$ & 6001200 & 108,109 \\
\hline V417 Cen & 141559.744 & -615349.53 & 312.71 & -0.64 & $20.25 / 04 / 95$ & 30 & $30.06 / 05 / 96$ & 60600 & 110,111 \\
\hline V748 Cen & 145936.679 & -332503.97 & 331.51 & +22.24 & $30.09 / 05 / 96$ & 60600 & & & 112 \\
\hline HD 149427 & 163742.613 & -554226.21 & 331.13 & -5.77 & $01.27 / 06 / 96$ & 1030210 & & & 113 \\
\hline V1017 Sgr & 183204.167 & -292312.64 & 4.48 & -9.11 & $28.43 / 05 / 96$ & 600 & & & 114 \\
\hline AS 325 & 185003.570 & -262415.38 & 8.96 & -11.42 & $19.01 / 10 / 94$ & 30180 & & & 115 \\
\hline
\end{tabular}

Asiago $1.82 \mathrm{~m}+B \& C+C C D, \quad 3400-7700 \AA, \quad 7.5 \AA /$ pix spectra

validated symbiotic stars:

\begin{tabular}{llllllllll} 
EG And & 004437.1869 & +404045.707 & 121.54 & -22.17 & $15.02 / 10 / 95$ & 41120 & $07.77 / 02 / 96$ & 103090 & 116,117 \\
AX Per & 013622.693 & +541502.29 & 129.53 & -8.04 & $15.03 / 10 / 95$ & 2040 & $07.78 / 02 / 96$ & 90300 & 118,119 \\
V471 Per & 015849.661 & +525348.59 & 133.12 & -8.64 & $15.04 / 10 / 95$ & 20180350 & $07.80 / 02 / 96$ & 90900 & 120,121 \\
BD Cam & 034209.3250 & +631300.501 & 140.84 & +6.44 & $15.91 / 10 / 95$ & 13 & $07.82 / 02 / 96$ & 134 & 122,123 \\
UV Aur & 052148.881 & +323043.15 & 174.22 & -2.35 & $15.91 / 10 / 95$ & 1090 & $07.81 / 02 / 96$ & 154590 & 124,125 \\
BX Mon & 072522.777 & -033550.76 & 220.04 & +5.88 & $10.81 / 03 / 95$ & 60250 & $07.85 / 02 / 96$ & 60240 & 126,127 \\
V694 Mon & 072551.289 & -074408.09 & 223.76 & +4.05 & $07.86 / 02 / 96$ & 60240 & & 128 \\
NQ Gem & 073154.5207 & +243012.567 & 194.63 & +19.35 & $10.80 / 03 / 95$ & 1030150 & $07.84 / 02 / 96$ & 3090 & 129,130 \\
RW Hya & 133418.133 & -252248.89 & 314.99 & +36.49 & $11.08 / 03 / 95$ & 2060200 & & 131 \\
IV Vir & 141634.310 & -214550.21 & 327.88 & +36.95 & $11.09 / 03 / 95$ & 20300 & & 132 \\
\hline
\end{tabular}


Table 1. continued.

\begin{tabular}{|c|c|c|c|c|c|c|c|c|c|}
\hline Name & $\begin{array}{l}\alpha_{J 2000} \\
\mathrm{~h} \quad \mathrm{~m} s\end{array}$ & $\begin{array}{l}\delta_{J 2000} \\
0,{ }^{\prime},\end{array}$ & $l\left(^{\circ}\right)$ & $b\left(^{\circ}\right)$ & obs. date & $\begin{array}{l}\text { expt } \\
\text { (s) }\end{array}$ & obs. date & $\begin{array}{l}\text { expt } \\
\text { (s) }\end{array}$ & $\begin{array}{l}\text { Fig. } \\
\text { N. }\end{array}$ \\
\hline $\mathrm{T} \mathrm{CrB}$ & 155930.1611 & +255512.601 & 42.37 & +48.16 & $\begin{array}{l}27.82 / 07 / 93 \\
15.76 / 10 / 95\end{array}$ & $\begin{array}{l}25180480 \\
30120\end{array}$ & $\begin{array}{l}10.99 / 03 / 95 \\
08.08 / 02 / 96\end{array}$ & $\begin{array}{l}120180 \\
90190320\end{array}$ & $\begin{array}{l}133,134 \\
135,136\end{array}$ \\
\hline AG Dra & 160141.0135 & +664810.139 & 100.29 & +40.97 & $\begin{array}{l}11.13 / 03 / 95 \\
08.06 / 02 / 96\end{array}$ & $\begin{array}{l}20300 \\
103045\end{array}$ & $15.77 / 10 / 95$ & 340 & $\begin{array}{l}137,138 \\
139\end{array}$ \\
\hline Hen 3-1341 & 170836.556 & -172630.19 & 5.02 & +13.39 & $27.84 / 07 / 93$ & 40900 & & & 140 \\
\hline Draco C-1 & 171957.655 & +575005.68 & 86.27 & +34.76 & $12.90 / 08 / 90$ & 7200 & $10.86 / 07 / 96$ & 8553600 & 141,142 \\
\hline M 1-21 & 173417.220 & -190922.87 & 6.96 & +7.36 & 27.87/07/93 & 600 & & & 143 \\
\hline RT Ser & 173951.993 & -115638.22 & 13.89 & +9.97 & 27.88/07/93 & 360 & & & 144 \\
\hline Pt 1 & 173849.552 & -235405.43 & 3.48 & +3.94 & 27.85/07/93 & 900 & & & 145 \\
\hline RS Oph & 175013.165 & -064228.47 & 19.80 & +10.37 & 27.88/07/93 & 30600 & $15.72 / 10 / 95$ & 20120 & 146,147 \\
\hline V343 Ser & 181222.153 & -114007.17 & 18.08 & +3.20 & 27.89/07/93 & 600 & 15.73/10/95 & 60420 & 148,149 \\
\hline FG Ser & 181507.096 & -001852.21 & 28.48 & +7.93 & $\begin{array}{l}11.20 / 03 / 95 \\
10.94 / 07 / 96\end{array}$ & $\begin{array}{l}300 \\
600\end{array}$ & $14.74 / 10 / 95$ & 60600 & $\begin{array}{l}150,151 \\
152\end{array}$ \\
\hline YY Her & 181434.207 & +205921.02 & 48.14 & +17.24 & $11.09 / 03 / 95$ & 60420 & $14.75 / 10 / 95$ & 60600 & 153,154 \\
\hline V443 Her & 182207.849 & +232719.96 & 51.23 & +16.59 & $\begin{array}{l}11.10 / 03 / 95 \\
15.78 / 10 / 95\end{array}$ & $\begin{array}{l}20300 \\
20120\end{array}$ & $14.85 / 10 / 95$ & 60120 & $\begin{array}{l}155,156 \\
157\end{array}$ \\
\hline MWC 960 & 184755.858 & -200551.13 & 14.53 & -8.27 & 27.90/07/93 & 30900 & & & 158 \\
\hline AS 327 & 185316.673 & -242259.29 & 11.14 & -11.23 & 27.93/07/93 & 30600 & & & 159 \\
\hline FN Sgr & 185354.776 & -185939.86 & 16.15 & -9.06 & 27.94/07/93 & 30900 & & & 160 \\
\hline Pe 2-16 & 185410.11 & -043853.9 & 29.10 & -2.72 & 27.96/07/93 & 900 & 15.74/10/95 & 60720 & 161,162 \\
\hline CM Aql & 190335.124 & $\begin{array}{llll}-03 & 03 & 14.28\end{array}$ & 31.59 & -4.09 & $\begin{array}{l}27.98 / 07 / 93 \\
15.75 / 10 / 95\end{array}$ & $\begin{array}{l}30900 \\
45900\end{array}$ & $11.20 / 03 / 95$ & 180 & $\begin{array}{l}163,164 \\
165\end{array}$ \\
\hline V919 Sgr & 190345.130 & -165955.19 & 19.01 & -10.32 & 27.95/07/93 & 30900 & & & 166 \\
\hline V1413 Aql & 190351.685 & +162831.74 & 49.02 & +4.77 & $\begin{array}{l}11.18 / 03 / 95 \\
15.79 / 10 / 95\end{array}$ & $\begin{array}{l}30240 \\
30300\end{array}$ & $14.86 / 10 / 95$ & 30240 & $\begin{array}{l}167,168 \\
169\end{array}$ \\
\hline Ap 3-1 & 191036.126 & +024928.69 & 37.64 & -2.97 & $14.82 / 10 / 95$ & 1803600 & & & 170 \\
\hline BF Cyg & 192353.506 & +294029.22 & 62.93 & +6.70 & $11.14 / 03 / 95$ & 60420 & $14.87 / 10 / 95$ & 60300 & 171,172 \\
\hline CH Cyg & 192433.0681 & +501429.128 & 81.86 & +15.58 & $\begin{array}{l}11.13 / 03 / 95 \\
11.05 / 07 / 96\end{array}$ & $\begin{array}{l}61575 \\
1030\end{array}$ & $14.96 / 10 / 95$ & 513 & $\begin{array}{l}173,174 \\
175\end{array}$ \\
\hline HM Sge & 194157.083 & +164439.92 & 53.57 & -3.15 & $14.88 / 10 / 95$ & 20150 & & & 176 \\
\hline QW Sge & 194549.54 & +183647.6 & 55.65 & -3.02 & $\begin{array}{l}11.17 / 03 / 95 \\
11.04 / 07 / 96\end{array}$ & $\begin{array}{l}30600 \\
60180300\end{array}$ & $14.88 / 10 / 95$ & 60600 & $\begin{array}{l}177,178 \\
179\end{array}$ \\
\hline CI Cyg & 195011.8339 & +354103.003 & 70.90 & +4.74 & $11.16 / 03 / 95$ & 30150 & $14.92 / 10 / 95$ & 20120 & 180,181 \\
\hline V1016 Cyg & 195705.027 & +394936.13 & 75.17 & +5.68 & $11.16 / 03 / 95$ & 630150 & 14.93/10/95 & 1075 & 182,183 \\
\hline PU Vul & 202113.330 & +213419.31 & 62.58 & -8.52 & $\begin{array}{l}11.18 / 03 / 95 \\
10.95 / 07 / 96\end{array}$ & $\begin{array}{l}15240 \\
102090\end{array}$ & $14.89 / 10 / 95$ & 15240 & $\begin{array}{l}184,185 \\
186\end{array}$ \\
\hline LT Del & 203557.244 & +201127.60 & 63.40 & -12.15 & $\begin{array}{l}11.19 / 03 / 95 \\
10.95 / 07 / 96\end{array}$ & $\begin{array}{l}300 \\
600\end{array}$ & $14.91 / 10 / 95$ & 60600 & $\begin{array}{l}187,188 \\
189\end{array}$ \\
\hline Hen 2-468 & 204118.991 & +344452.33 & 75.94 & -4.44 & $14.93 / 10 / 95$ & 901200 & $10.96 / 07 / 96$ & 900 & 190,191 \\
\hline V1329 Cyg & 205101.27 & +353454.1 & 77.80 & -5.56 & $14.97 / 10 / 95$ & 30163 & $11.01 / 07 / 96$ & 60120 & 192,193 \\
\hline V407 Cyg & 210209.84 & +454632.6 & 86.99 & -0.49 & $11.21 / 03 / 95$ & 30240 & $14.98 / 10 / 95$ & 30250500 & 194,195 \\
\hline AG Peg & 215101.9749 & +123732.113 & 69.28 & -30.89 & $14.91 / 10 / 95$ & 536 & $11.00 / 07 / 96$ & 20 & 196, 197 \\
\hline $\mathrm{Z}$ And & 233339.9505 & +484905.947 & 109.98 & -12.09 & $15.01 / 10 / 95$ & 1022 & $07.73 / 02 / 96$ & 60600 & 198,199 \\
\hline R Aqr & 234349.4616 & -151704.202 & 66.52 & -70.33 & $14.90 / 10 / 95$ & 2090180 & & & 200 \\
\hline & & . & sуiाl & a & 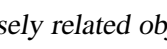 & & & & \\
\hline V641 Cas & 000926.339 & +635714.13 & 118.34 & +1.46 & $15.88 / 10 / 95$ & 530 & $07.76 / 02 / 96$ & 30180 & 201,202 \\
\hline GH Gem & 070412.801 & +120334.26 & 203.57 & +8.23 & $10.96 / 03 / 95$ & 6001200 & & & 203 \\
\hline ZZ CMi & 072413.9987 & +085351.777 & 208.64 & +11.30 & $10.79 / 03 / 95$ & 30120700 & $07.84 / 02 / 96$ & 3090 & 204,205 \\
\hline TX CVn & 124442.065 & +364550.68 & 130.93 & +80.26 & $10.98 / 03 / 95$ & 180200 & 08.03/02/96 & 90360600 & 206, 207 \\
\hline V503 Her & 173640.461 & +2318 12.01 & 47.00 & +26.23 & $15.77 / 10 / 95$ & 60300 & & & 208 \\
\hline XX Oph & 174356.4972 & -061608.750 & 19.41 & +11.95 & $15.72 / 10 / 95$ & 1070 & & & 209 \\
\hline V335 Vul & 192314.108 & +242739.97 & 58.22 & +4.40 & 24.72/11/93 & 60360 & 21.72/11/97 & 30300 & 210,211 \\
\hline Hen 2-442 & 193943.36 & +262933.0 & 61.80 & +2.13 & $15.81 / 10 / 95$ & 901200 & & & 212 \\
\hline OY Cyg & 195443.859 & +391757.85 & 74.48 & +5.80 & $15.83 / 10 / 95$ & 60420 & & & 213 \\
\hline V627 Cas & 225740.967 & +584912.49 & 108.66 & -0.86 & $14.73 / 10 / 95$ & 30300 & $07.74 / 02 / 96$ & 600 & 214,215 \\
\hline V630 Cas & 234851.956 & +512739.32 & 113.10 & -10.21 & $15.86 / 10 / 95$ & 60420 & & & 216 \\
\hline
\end{tabular}


- the rich emission line spectrum of symbiotic stars will be studied in a global approach;

- properties of the cool giants will be derived from the absorption spectrum in the red region.

\section{The data}

The list of program stars and the journal of observations is given in Table 1. The number of symbiotic stars (validated and probable) observed for this atlas is 130, with a total of 213 spectra presented in Figs. 4-216. Spectra of 40 reference stars are given in Figs. 217-256, with Table 2 listing their relevant properties. Figure 1 gives an example of Figs. 4-256 available electronically only. Of the 90 symbiotic stars observed from ESO, 22 have been re-observed about one year later. Fifty-five are instead the symbiotics observed from Asiago, 21 of which were re-observed once, 9 twice and 1 three times over a two and a half year period. For some systems our observations document the spectral changes induced by an outburst state, as for CL Sco (during the 1995 quiescence in Fig. 49, and at 1996 outburst in Fig. 50), or their interplay with the pulsation activity of the cool giant, as for the carbon symbiotic Mira UV Aur (at Mira's minimum in Fig. 124, and at Mira's maximum in Fig. 125).

The program stars have been selected among those listed in Allen's (1984) catalogue and those discovered later (and summarized in the new catalogue of symbiotic stars by Belczynski et al. 2000), including objects from the Meyssonnier \& Azzopardi (1993) survey of SMC.

The object names (as given in Table 1 and the first column of Table 3) are sometimes different from those used in the literature, because either (i) a variable star name has been assigned since (for example V1413 Aql for AS 338), or (ii) there have been re-organizations in the name coding within SIMBAD (for example, He 2-468 was replaced by Hen 2-468, or Hen 1591 is now Hen 3-1591). A list of correspondences is given in Table 3, where Col. 2 contains Allen (1984) names for the systems renamed subsequently.

The spectrophotometric observations have been performed with B\&C + CCD spectrographs at ESO and Asiago. All spectra have been reduced with the IRAF software package. Standard procedures (including bias and flat field correction, wavelength and flux calibration) have been applied. Cosmic rays on the stellar tracing were cleaned manually on the extracted spectrum after inspection of the original two dimensional frames. For the vast majority of the program stars we have obtained more than one spectrum per night (with the aim of appropriately exposing the continuum as well as avoiding saturation of the strongest emission lines), and their inter-comparison greatly assisted during the manual cleaning of cosmic-rays.

\subsection{Astrometry}

Historically inaccurate or erroneous coordinates of several symbiotic stars are still present in the current literature.
Moreover, correspondence between symbiotic stars and entries in astrometric catalogues has not been systematically explored yet. The latter is an important task because, once the correspondence is established, coordinates of symbiotic stars will be automatically improved every time the catalogues are re-calibrated (like USNO-A1 that has been recalibrated into USNO-A2 when the Hipparcos/Tycho reference stars have become available) or cross-referenced toward newer and higher precision catalogues.

Correspondence with sources in astrometric catalogues is established in Table 3 (last column) for all the program stars. Help has been provided by the Aladin graphical interface at CDS (http://aladin.u-strasbg.fr/aladin.gml) that allows overplotting of astrometric catalogues over the digitized DSS-I and DSS-II plates. The astrometric identification has been searched for and taken from the following catalogues, in order of preference: Hipparcos, Tycho-2, USNO-A2.0, GSC-1, 2MASS. Only 6 objects lack any astrometric identification, while 14 and 24 objects are included in the Hipparcos and Tycho-2 catalogues, respectively.

Coordinates of program stars in Table 1 have not been adapted or re-processed from the existing literature, but re-compiled from scratch. Hender \& Munari (2000, 2001) give astrometrically measured accurate positions for 40 of our program stars (linked to the USNO-A2.0 reference system). For the remaining targets the positions as given in the appropriate astrometric catalogues (following identifications in Table 3) have been adopted. For the 6 objects without an entry in the surveyed astrometric catalogues, coordinates have been measured by us on the digitized DSS-II plates (or, if not available, on the DSS-I ones). All coordinates are on J2000.0 equinox, but epochs are those of the corresponding catalogues (this should not be a major concern given the minimal proper motions typical for most symbiotic stars).

\subsection{ESO observations}

The ESO observations have been performed with the $\mathrm{B} \& \mathrm{C}+\mathrm{CCD}$ spectrograph at the $1.5 \mathrm{~m}$ telescope under photometric conditions (sparse data from non optimal nights have not been used in the present atlas). We used a $400 \mathrm{l} / \mathrm{mm}$ grating (\# 25) and a 2 arcsec slit, always aligned along the parallactic angle for pointings at zenital distances larger than $35^{\circ}$. The CCD was a $2048 \times 2048,15 \mu \mathrm{m}$ size, thinned and back-illuminated to enhance UV sensitivity. The dispersion was $\sim 2.5 \AA /$ pix, with a $F W H M(\mathrm{PSF}) \sim 2$ pixels. The covered spectral range changes somewhat from one observing run to another, with the extremes of 3200-8900 and 3400-9100 $\AA$. The seeing during the observing nights was always smaller than the slit width (as can be derived from the simultaneous absolute measurements with the La Silla Meteo Monitor and from the measured FWHM perpendicular to the dispersion on the recorded stellar spectra). For a 


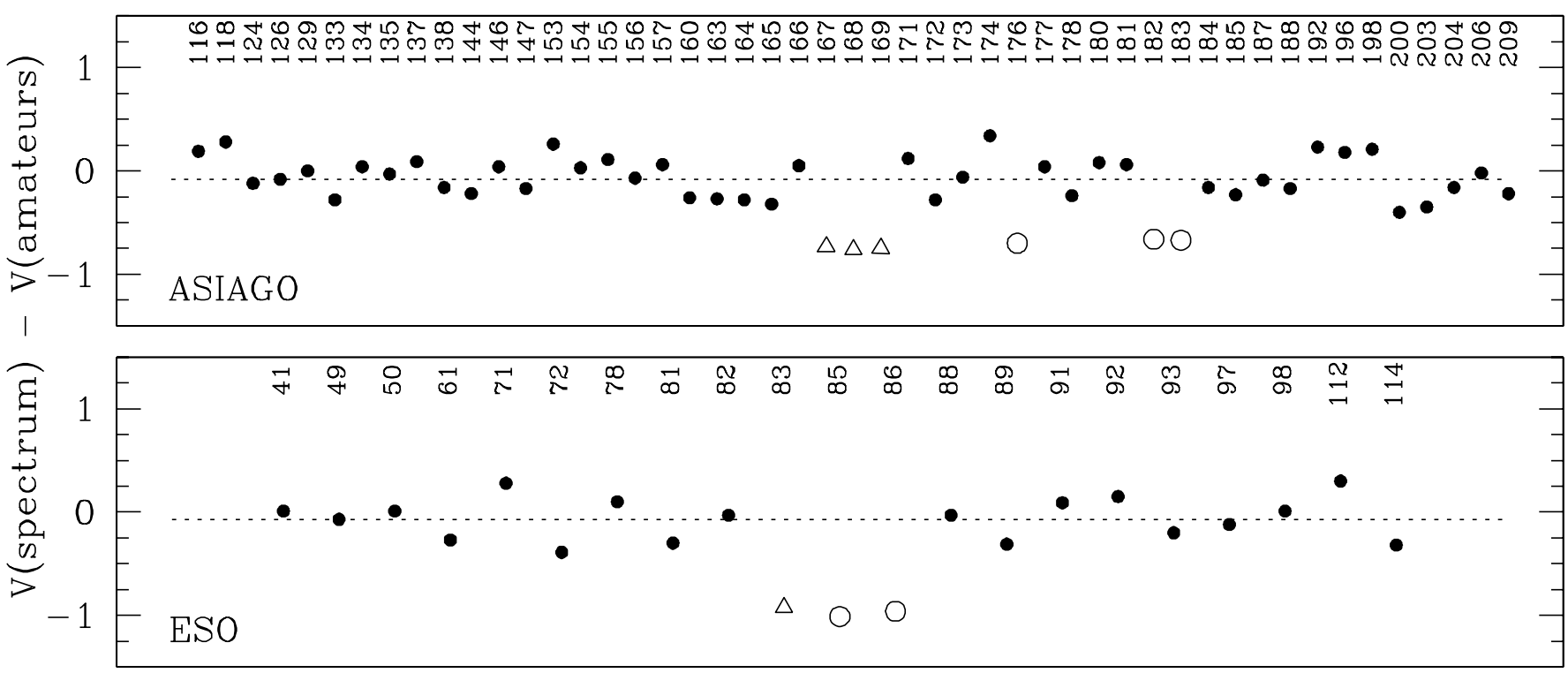

Fig. 2. Comparison of $V$ magnitudes of symbiotic stars as estimated by amateur astronomers (VSNET, VSOLJ, AFOEV databases) and as derived from flux calibrated spectra in this atlas. To identify the given symbiotic star and its particular spectrum, the upper row in each panel lists the corresponding figure number (cf. Table 1). See text for details (Sect. 2.5).

non-marginal part of the time the seeing was better than 1 arcsec. Several spectrophotometric standards were observed each night in order to achieve absolute flux calibration and monitor stable transparency conditions.

\subsection{Asiago observations}

The Asiago observations were secured with the Boller \& Chivens spectrograph attached to the $1.82 \mathrm{~m}$ telescope operated by Osservatorio Astronomico di Padova atop of Mount Ekar (Asiago, Italy), during nights of suitable photometric conditions. The detector was a Thompson TH7882 UV-coated CCD, $580 \times 388$ pixels of $23 \mu \mathrm{m}$ size. We used a $150 \mathrm{ln} / \mathrm{mm}$ grating giving a dispersion of $7.5 \AA /$ pixel, generally covering the wavelengths 3350-7550 $\AA$ (exact limits variable according to the observing runs). The slit width was $\sim 2.0$ arcsec, giving a $F H W M(\mathrm{PSF}) \sim 2$ pixels. When the object's zenith distance exceeded $45^{\circ}$, the slit was aligned along the parallactic angle. Each night at least four spectrophotometric standard stars were observed more than once at different airmasses for flux calibration.

\subsection{Reference objects}

A number of spectra of reference objects have been secured with the same instrumentation during symbiotic star observing runs. The reference objects include MKK cool giant standards, Miras, planetary nebulae, Wolf Rayet stars, white dwarfs, hot sub-dwarf, classical novae, and are intended to assist inspection and interpretation of symbiotic star spectra. They are listed in Table 2 and their spectra are presented in Figs. 217-256.

\subsection{Flux accuracy}

It appears appropriate to quantify the accuracy of absolute fluxes in this atlas. For symbiotic stars no optical photometry was carried out simultaneously with the spectroscopic observations. However, a number of program symbiotic stars are regularly observed by several organizations of amateur astronomers. We have consulted the on-line databases of VSNET, VSOLJ and AFOEV and found useful data to support comparison with 70 of the spectra presented in this atlas (49 Asiago spectra and 21 ESO spectra, reflecting the predominance of amateurs in the northern hemisphere and higher brightness of average symbiotic stars known in the northern hemisphere). When possible, the amateurs' lightcurves have been interpolated/extrapolated to derive the visual magnitude for the date of the given spectral observation. In a few cases an estimate was found in the amateurs' archives for the exact date of the spectral observations. Such estimates have been adopted unless an eye inspection of the whole light-curve rendered them unreliable.

The $V$ magnitude was derived from our spectra by convolving them with the $V$ band-pass profile (taken from Ažusienis \& Straižys 1969). The comparison between the spectroscopic and amateurs' $V$ magnitudes is presented in Fig. 2 separately for the Asiago and ESO data. The comparison gives for the ESO spectra:

$V_{\text {spectrum }}-V_{\text {amateurs }}=-0.06 ; \quad \sigma=0.21$

and similarly for the Asiago spectra:

$V_{\text {spectrum }}-V_{\text {amateurs }}=-0.05 ; \quad \sigma=0.19$.

The offset is small and can be easily accounted for by a number of possibilities, the first and the most obvious one 

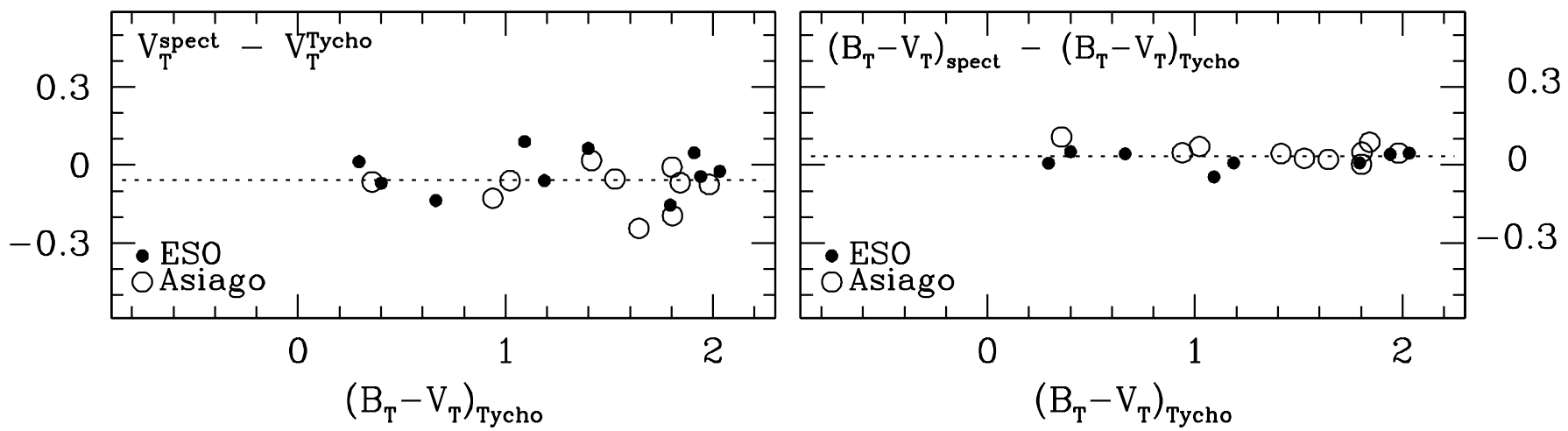

Fig. 3. Comparison of $V_{\mathrm{T}}$ magnitudes and $\left(B_{\mathrm{T}}-V_{\mathrm{T}}\right)$ colors of template stars in Table 2 as measured by Hipparcos/Tycho and as derived from spectra in this atlas. See text for details (Sect. 2.5).

being the difference between the eye response and the $V$ band profile.

ESO and Asiago observations appear equally accurate, as seen in Figs. 2 and 3, with an average 0.20 mag scatter that deserves some comment. Several mechanisms contribute to it, including $[a]$ errors in the comparison sequences used by amateurs $\left(\sigma_{\mathrm{cs}}\right),[b]$ errors in estimating the magnitude by the amateurs and uncertainties in interpolating/extrapolating their lightcurve to the desired date $\left(\sigma_{\text {est }}\right),[c]$ day-by-day random variability of symbiotic stars $\left(\sigma_{\text {var }}\right)$, and obviously $[d]$ the errors in fluxing our spectra $\left(\sigma_{\text {flux }}\right)$. Because these errors are unrelated and follow normal distributions we write

$\sigma^{2}=(0.20)^{2}=\sigma_{\mathrm{cs}}^{2}+\sigma_{\text {est }}^{2}+\sigma_{\text {var }}^{2}+\sigma_{\text {flux }}^{2}$.

Based on common experience it may be assumed that $\sigma_{\mathrm{cs}} \geq 0.05, \sigma_{\text {est }} \geq 0.15$, and $\sigma_{\text {var }} \sim 0.1\left(\sigma_{\mathrm{cs}}=0.05\right.$ is obviously an underestimate, at least because magnitudes of comparison stars on amateur finding charts are given to one decimal figure only). So $\sigma_{\text {flux }} \leq 6 \%$, i.e. the fluxes of each individual spectrum are on the average accurate better than $6 \%$. This is in fair agreement with results of inter-calibrations of spectrophotometric standard stars observed every night that suggest an accuracy of $5 \%$ or better.

Certain points in Fig. 3 are marked with open circles and triangles. The open circles refer to HM Sge and V1016 Cyg that have spectra resembling planetary nebulae. Minimal differences between the $V$ band and eye response curves around the immensely strong $\mathrm{H} \alpha$ and [OIII] lines fully account for the $\sim 0.7 \mathrm{mag}$ systematic difference of both Asiago and ESO spectra versus amateurs' estimates. The open triangles refer to V1413 Aql that shows the same $\sim 0.8 \mathrm{mag}$ systematic difference between the Asiago and ESO spectra vs. amateurs' estimates: the reason may be an error in the comparison sequence around V1413 Aql used by the amateurs.

Another independent way to estimate the flux accuracy is offered by the spectra of the reference objects listed in Table 2. Selecting those not carrying a variable star name among those observed by Hipparcos/Tycho, we found

$$
\begin{aligned}
& V_{\mathrm{T}}^{\text {spectra }}-V_{\mathrm{T}}^{\text {Tycho }}=-0.06 ; \quad \sigma=0.08 \\
& (B-V)_{\mathrm{T}}^{\text {spectra }}-(B-V)_{\mathrm{T}}^{\text {Tycho }}=+0.03 ; \quad \sigma=0.03 .
\end{aligned}
$$

Here the spectra were convolved with the $B_{\mathrm{T}}$ and $V_{\mathrm{T}}$ band profiles as given in the Hipparcos Catalogue (ESA SP-1200, June 1997 , p. 42; slightly modified transmission profiles are suggested by Bessell 2000). The uncertainties in the shutter aperture time $(\sim 0.1 \mathrm{~s}$ in both Asiago and ESO observations) become notable in the observations of the bright template stars in Table 2 with an average exposure time of only $2.5 \mathrm{~s}$. Also, non-perfect placement of such bright objets on the slit, which is compensated for faint objects by guiding, may contribute to the scatter in Eq. (5). Finally, it is worth remembering that Tycho data for bright stars are accurate to $0.012 \mathrm{mag}$ in $V_{\mathrm{T}}$ and $0.02 \mathrm{mag}$ in $(B-V)_{\mathrm{T}}$.

In light of these considerations it seems safe to argue that the fluxes in this atlas are generally correct within

$\sigma_{\text {flux }} \lesssim 5 \%$

at least for the $B, V$ and $R$ band wavelength ranges. A lower accuracy is probable for faint red objects at $\lambda \leq$ $3800 \AA$ or for pure emission line spectra at $\lambda \geq 8700 \AA$.

\section{The atlas}

Each spectral observation listed in Tables 1 and 2 is presented by a separate figure. The format of the figures is identical to Fig. 1. UT dates are in the DD.dd/MM/YY format.

At upper right a full-scale, compressed view of the whole recorded spectrum is given, and the units for absolute fluxes are indicated. The same units are valid for the other panels that present zoomed-in portions of the spectrum.

For ESO spectra the first zoomed-in panel always runs from 3200 to $5250 \AA$, the second from 5150 to $7200 \AA$, and the third from 7100 to $9150 \AA$. For Asiago spectra, 
given the shorter wavelength range covered, two zoomedin panels suffice, the first from 3250 to $5600 \AA$, the second from 5350 to $7600 \AA$.

Stronger emission lines are generally truncated in the zoomed-in panels to emphasize the visibility of finer details. The full height of emission lines can be read from the compressed view of the whole spectrum at the upper right.

\section{References}

Acker, A., Lundstrom, I., \& Stenholm, B. 1988, A\&AS, 73, 325 Ažusienis, A., \& Strayžys, V. 1969, Sov. Astron., 13, 316

Allen, D. A. 1984, Proc.A.S.A., 5, 369

Belczynski, K., Mikolajewska, J., Munari, U., Ivison, R. J., \& Friedjung, M. 2000, A\&AS, 146, 407

Bessell, M. S. 2000, PASP, 112, 961

Blair, W. P., Feibelman, W. A., Michalitsianos, A. G., \& Stencel, R. E. 1983, ApJS, 53, 573

Gutiérrez-Moreno, A., Moreno, H., \& Costa, E. 1999, PASP, 111,571
Ipatov, A. P., \& Yudin, B. F. 1986, A\&AS, 65, 51

Ivison, R. J., Bode, M. F., \& Meaburn, J. 1994, A\&AS, 103 201

Henden, A., \& Munari, U. 2000, A\&AS, 143, 343

Henden, A., \& Munari, U. 2001, A\&A, 372, 145

Henden, A., \& Munari, U. 2002, A\&A, to be submitted

Kenyon, S. J. 1986, The symbiotic stars (Cambridge University Press)

Kenyon, S. J., \& Fernandez-Castro, T. 1987, AJ, 93, 938

Meyssonnier, N., \& Azzopardi, M. 1993, A\&AS, 102, 451

Medina Tanco, G. A., \& Steiner, J. E. 1995, AJ, 109, 1770

Meier, S. R., Kafatos, M., Fahey, R. P., \& Michalitsianos, A. G. 1994, ApJS, 94, 183

Mürset, U., Schild, H., \& Vogel, M. 1996, A\&A, 307, 516

Pereira, C. B., de Araújo, F. X., \& Landaberry, S. 1999, MNRAS, 309, 1074

Schild, H., Boyle, S. J., \& Schmid, H. M. 1992, MNRAS, 258, 95

Schmid, H. M., \& Schild, H. 1994, A\&A, 281, 145

Schulte-Ladbeck, R. E. 1988, A\&A, 189, 97

Van Winckel, H., Duerbeck, H. W., \& Schwarz, H. E. 1993, A\&AS, 102, 401 